\title{
SOME MARX-STROHHÄCKER TYPE RESULTS FOR A CLASS OF MULTIVALENT FUNCTIONS
}

\section{NUNOKAWA, H. M. SRIVASTAVA, N. TUNESKI, AND B. JOLEVSKA-TUNESKA}

Received 08 April, 2016

\begin{abstract}
In this paper, two Marx-Strohhäcker type results are proven for a certain class of analytic and multivalent functions in the open unit disk $\mathbb{D}$. The first result gives the order of multivalent starlikeness for multivalently convex functions of some specified order. The second result provides a lower bound over the unit disk $\mathbb{D}$ of $\Re\left(\frac{f(z)}{z^{p}}\right)$ for functions $f(z)$ that are multivalently starlike of a given order. Relevant connections of the results presented here with earlier results are also indicated.
\end{abstract}

2010 Mathematics Subject Classification: 30C45; 30C50

Keywords: analytic functions, multivalent functions, Marx-Strohhäcker type results, Jack's problem, principle of subordination

\section{INTRODUCTION, DEFINITIONS AND PRELIMINARIES}

Let $\mathscr{H}(\mathbb{D})$ denote the class of all functions which are analytic in the open unit disk

$$
\mathbb{D}=\{z: z \in \mathbb{C} \text { and }|z|<1\} .
$$

For $n \in \mathbb{N}(\mathbb{N}:=\{1,2,3, \cdots\})$ and $a \in \mathbb{C}$, let

$$
\mathscr{H}[a, n]=\left\{f: f \in \mathscr{H}(\mathbb{D}) \text { and } f(z)=a+a_{n} z^{n}+a_{n+1} z^{n+1}+\cdots\right\} .
$$

We also let $\mathcal{A}_{p}(p \in \mathbb{N})$ be the subclass of $\mathscr{H}(\mathbb{D})$ consisting of functions of the following normalized form:

$$
f(z)=z^{p}+a_{p+1} z^{p+1}+a_{p+2} z^{p+2}+\cdots
$$

and write $\mathcal{A} \equiv \mathcal{A}_{1}$. The functions (mappings) in $\delta \subset \mathcal{A}$ are one-to-one and are called normalized univalent functions in $\mathbb{D}$. For more details about analytic, univalent and multivalent functions, see (for example) [1,5,9].

The classes of $p$-valently starlike functions of order $\alpha$ in $\mathbb{D}$ and $p$-valently convex functions of order $\alpha$ in $\mathbb{D}(p \in \mathbb{N} ; 0 \leqq \alpha<p)$ are defined by

$$
\delta_{p}^{*}(\alpha)=\left\{f \in \mathcal{A}_{p}: \Re\left(\frac{z f^{\prime}(z)}{f(z)}\right)>\alpha \quad(z \in \mathbb{D})\right\}
$$

(C) 2017 Miskolc University Press 
and

$$
\mathcal{K}_{p}(\alpha)=\left\{f \in \mathcal{A}_{p}: \Re\left(1+\frac{z f^{\prime \prime}(z)}{f^{\prime}(z)}\right)>\alpha \quad(z \in \mathbb{D})\right\},
$$

respectively. In the case when $p=1$, we respectively have the usual classes $\delta^{*}(\alpha)$ and $\mathcal{K}(\alpha)$ of starlike and convex functions of order $\alpha$ in $\mathbb{D}$. Moreover, in the case when $p \in \mathbb{N} \backslash\{1\}$, we are led to $p$-valently analytic function classes which correspond essentially to the classes $\delta_{p}^{*}$ and $\mathcal{K}_{p}$ of $p$-valently starlike and $p$-valently convex functions of order $\alpha$ in $\mathbb{D}$. For simplicity, in the case when $\alpha=0$, we will use the notations $\delta_{p}^{*}$ and $\mathcal{K}_{p}$ and write

$$
\varsigma^{*}=\gamma_{1}^{*}=\gamma_{1}^{*}(0) \quad \text { and } \quad \mathcal{K}=\mathcal{K}_{1}=\mathcal{K}_{1}(0)
$$

in the case when $\alpha=0$ and $p=1$.

It is a well-known result due to Marx and Strohhäcker $[4,11]$ that convex functions have the order $\frac{1}{2}$ of starlikeness, that is, for $f \in \mathcal{A}$, we have

$$
\Re\left(1+\frac{z f^{\prime \prime}(z)}{f^{\prime}(z)}\right)>0 \quad(z \in \mathbb{D}) \quad \Longrightarrow \quad \Re\left(\frac{z f^{\prime}(z)}{f(z)}\right)>\frac{1}{2} \quad(z \in \mathbb{D}) .
$$

The function $f(z)=\frac{z}{1-z}$ shows that this result is sharp, that is, the number $\frac{1}{2}$ cannot be replaced by a larger one. Let us call this the Marx-Strohhäcker result of Type I. It was proven in [6] that this implication is not true for $p$-valent functions $(p \geqq 2)$. Namely, it was proven that, if $p \in \mathbb{N} \backslash\{1\}$, then there exists $f \in \mathcal{K}_{p}$ such that $f \in \mathcal{S}_{p}^{*}$, but $f \notin 8_{p}^{*}(\alpha)$ for all $\alpha>0$, that is, there exists $f \in \mathcal{A}_{p}$ such that

$$
\Re\left(1+\frac{z f^{\prime \prime}(z)}{f^{\prime}(z)}\right)>0 \quad(z \in \mathbb{D}) \quad \text { and } \quad \Re\left(\frac{z f^{\prime}(z)}{f(z)}\right)>0 \quad(z \in \mathbb{D}),
$$

but the following inequality:

$$
\Re\left[\frac{z f^{\prime}(z)}{f(z)}\right]>\alpha \quad\left(z_{0} \in \mathbb{D}\right)
$$

is not satisfied for any $\alpha>0$.

In view of the above observation, it is natural to ask the following question: If $f(z)$ is a $p$-valently convex function of order $\alpha$ in $\mathbb{D}(p \in \mathbb{N} \backslash\{1\} ; 0 \leqq \alpha<p)$, what is the order $\beta$ of $p$-valent starlikeness of the function $f(z)$ ? For the case when $\frac{p-1}{2} \leqq \alpha<p$, this problem is solved in a sharp way (that is, the best way possible) by Srivastava et al. [10], in which they showed that

$$
\mathcal{K}_{p}(\alpha) \subset \delta_{p}^{*}\left(\widehat{\beta}_{1}(\alpha, p)\right),
$$

where

$$
\widehat{\beta}_{1}(\alpha, p)=\frac{p}{{ }_{2} F_{1}\left(1,2(p-\alpha) ; p+1 ; \frac{1}{2}\right)}
$$

is the largest number with such property. In this paper, we extend this result to the case when $0 \leqq \alpha<\frac{p-1}{2}$. Unfortunately, so far we have not succeeded in proving 
sharpness of results which we have obtained in this investigation. There are indications that they are not sharp. However, finding their sharp versions remains an open problem.

The analogue problem for the class of univalent functions was posed by Jack [2]: What is the largest number $\beta=\beta(\alpha)$ so that $\mathcal{K}(\alpha) \subset \delta^{*}(\beta(\alpha))$ ? MacGregor [3] determined the exact value of $\beta(\alpha)$ for each $\alpha(0 \leqq \alpha<1)$ as the infimum over the disk $\mathbb{D}$ of the real part of a specific analytic function. It has been conjectured that this infimum is attained on the boundary of $\mathbb{D}$ at $z=-1$. Wilken and Feng in [12] asserted MacGregor's conjecture and obtained

$$
\widehat{\beta}_{2}(\alpha)= \begin{cases}\frac{1-2 \alpha}{2^{2(1-\alpha)}-2} & \left(\alpha \neq \frac{1}{2}\right) \\ \frac{1}{2 \ln 2} & \left(\alpha=\frac{1}{2}\right) .\end{cases}
$$

We will also give a generalization for the class of multivalent functions of another classical Marx-Strohhäcker result ([4, 11]):

$$
\Re\left(\frac{z f^{\prime}(z)}{f(z)}\right)>\frac{1}{2} \quad(z \in \mathbb{D}) \quad \Longrightarrow \quad \Re\left(\frac{f(z)}{z}\right)>\frac{1}{2} \quad(z \in \mathbb{D}) .
$$

Let us call this the Marx-Strohhäcker result of Type II. For several other investigations involving the Marx-Strohhäcker type results for other classes of multivalent functions, see (for example) the earlier works by Srivastava et al. [8, Section 3] and [7, Section 3]).

For proving the results in this paper, we will use the following Lemma from the theory of differential subordination between analytic functions.

Lemma 1. (see [5, p. 35, Theorem 2.3i(i)]). Let $\Omega \subset \mathbb{C}$ and suppose that the function $\psi: \mathbb{C}^{2} \times \mathbb{D} \rightarrow \mathbb{C}$ satisfies $\psi(i x, y ; z) \notin \Omega$ for all $x \in \mathbb{R}, y \leqq-\frac{n}{2}\left(1+x^{2}\right)$ and $z \in \mathbb{D}$. If $q \in \mathscr{H}[1, n]$ and $\psi\left(q(z), z q^{\prime}(z) ; z\right) \in \Omega$ for all $z \in \mathbb{D}$, then

$$
\Re(q(z))>0 \quad(z \in \mathbb{D}) .
$$

\section{A MARX-STROHHÄCKER RESUlT OF TYPE I}

Our Marx-Strohhäcker result of Type I is contained in Theorem 1 below.

Theorem 1. Let $p \in \mathbb{N}$ and $0 \leqq \beta<p$. Also let

$$
\alpha \equiv \alpha(\beta, p)= \begin{cases}\beta-\frac{1}{2}\left(\frac{\beta}{p-\beta}\right) & \left(0 \leqq \beta<\frac{p}{2}\right) \\ \beta-\frac{1}{2}\left(\frac{p-\beta}{\beta}\right) & \left(\frac{p}{2} \leqq \beta<p\right) .\end{cases}
$$


If $f \in \mathcal{A}_{p}$ and

$$
\Re\left(1+\frac{z f^{\prime \prime}(z)}{f^{\prime}(z)}\right)>\alpha \quad(z \in \mathbb{D}),
$$

then

$$
\Re\left(\frac{z f^{\prime}(z)}{f(z)}\right)>\beta \quad(z \in \mathbb{D})
$$

or, equivalently,

$$
\mathcal{K}_{p}(\alpha(\beta, p)) \subset \vartheta_{p}^{*}(\beta),
$$

that is, $p$-valently convex functions of order $\alpha(\beta, p)$ in $\mathbb{D}$ have the $\beta$ order of $p$-valent starlikeness in $\mathbb{D}$.

Proof. If we let the function $q(z)$ be given by

$$
q(z)=\frac{1}{p-\beta}\left(\frac{z f^{\prime}(z)}{f(z)}-\beta\right),
$$

then $q(z) \in \mathscr{H}[1,1]$. Further, for the function

$$
\psi(r, s ; z)=\frac{s(p-\beta)}{r(p-\beta)+\beta}+r(p-\beta)+\beta,
$$

we find by using (2.2) that

$$
\psi\left(q(z), z q^{\prime}(z) ; z\right)=1+\frac{z f^{\prime \prime}(z)}{f^{\prime}(z)} \in \Omega \equiv\{\omega: \Re \omega>\alpha\} \quad(z \in \mathbb{D}) .
$$

So, by the Lemma in Section 1, for proving (2.3) or, equivalently, that

$$
\Re(q(z))>0 \quad(z \in \mathbb{D}),
$$

it is enough to show that

$$
\psi(i x, y ; z)=\frac{y(p-\beta)}{i x(p-\beta)+\beta}+i x(p-\beta)+\beta \notin \Omega
$$

for all real $x, y \leqq-\frac{1+x^{2}}{2}(n=1$ in the Lemma in Section 1$)$ and for all $z \in \mathbb{D}$. Indeed, we have

$$
\begin{aligned}
\Re(\psi(i x, y ; z)) & \leqq \beta\left(1+\frac{-\left(\frac{1+x^{2}}{2}\right)(p-\beta)}{\beta^{2}+x^{2}(p-\beta)^{2}}\right) \\
& =\beta\left(1+\frac{1}{p-\beta} \cdot \frac{p\left(\beta-\frac{p}{2}\right)}{\beta^{2}+x^{2}(p-\beta)^{2}}-\frac{\frac{1}{2}}{p-\beta}\right) \equiv \varphi(\beta, p, x),
\end{aligned}
$$

and from

$$
\frac{\partial}{\partial x} \varphi(\beta, p, x)=\frac{\beta p(p-\beta)}{\left[\beta^{2}+x^{2}(p-\beta)^{2}\right]^{2}}(p-2 \beta) x
$$


we observe that $\varphi(\beta, p, x)$ is a piecewise monotone function of $x$, which increases for $x \geqq 0$ and decreases for $x<0$ when $0 \leqq \beta<\frac{p}{2}$ and which decreases for $x \geqq 0$ and increases for $x<0$ when $0 \leqq \beta<\frac{p}{2}$. So, we get

$$
\begin{aligned}
& \varphi(\beta, p, x) \leqq\left\{\begin{array}{cc}
\lim _{x \rightarrow \infty} \varphi(\beta, p, x)=\beta-\frac{1}{2}\left(\frac{\beta}{p-\beta}\right) & \left(0 \leqq \beta<\frac{p}{2}\right) \\
\varphi(\beta, p, 0)=\beta-\frac{1}{2}\left(\frac{p-\beta}{\beta}\right) & \left(\frac{p}{2} \leqq \beta<p\right)
\end{array}\right\} \\
& =\alpha(\beta, p) \text {. }
\end{aligned}
$$

This evidently completes the proof of Theorem 1 .

Remark 1. Each of the following observations is worth recording here.

(i) The value $\alpha(\beta, p)$ is well defined, that is, $0 \leqq \alpha(\beta, p)<p$. In the case when $p=1$, this can be directly verified. In the case when $p \in \mathbb{N} \backslash\{1\}$, it follows from the fact that $\alpha(\beta, p)$ is a piecewise strictly increasing function of $\beta$ on the interval $[0, p)$ (which will be shown in the proof of Theorem 2 below). Thus, clearly, we have

$$
0=\alpha(0, p) \leqq \alpha(\beta, p)<\lim _{\beta \rightarrow p} \alpha(\beta, p)=\beta<p .
$$

(ii) For $p=1$ and $\beta=\frac{1}{2}$ in Theorem 1, we obtain $\alpha=0$, that is, we obtain the Marx-Strohhäcker's result as in the implication (1.1).

Theorem 1 can be rewritten in the following equivalent form, which gives the Marx-Strohhäcker result of Type I for multivalent functions for any $\alpha \in[0, p)$.

Theorem 2. Let $p \in \mathbb{N}, 0 \leqq \alpha<p$ and

$$
\beta \equiv \beta(\alpha, p)= \begin{cases}\frac{2(\alpha+p)-1-\sqrt{[2(\alpha+p)-1]^{2}-16 \alpha p}}{4} & \left(0 \leqq \alpha<\frac{p-1}{2}\right) \\ \frac{2 \alpha-1+\sqrt{(2 \alpha-1)^{2}+8 p}}{4} & \left(\frac{p-1}{2} \leqq \alpha<p\right) .\end{cases}
$$

If $f \in \mathcal{A}_{p}$ and

$$
\Re\left(1+\frac{z f^{\prime \prime}(z)}{f^{\prime}(z)}\right)>\alpha \quad(z \in \mathbb{D}),
$$

then

$$
\Re\left(\frac{z f^{\prime}(z)}{f(z)}\right)>\beta \quad(z \in \mathbb{D})
$$

or, equivalently,

$$
\mathcal{K}_{p}(\alpha) \subset \gamma_{p}^{*}(\beta(\alpha, p)),
$$


that is, $p$-valently convex functions of order $\alpha$ have the $\beta(\alpha, p)$ order of $p$-valent starlikeness.

Proof. For the function $\alpha(\beta, p)$ defined by (2.1), we have

$$
\frac{\partial}{\partial \beta} \alpha(\beta, p)=\left\{\begin{array}{ll}
1-\frac{p}{2(p-\beta)^{2}} & \left(0<\beta<\frac{p}{2}\right) \\
1+\frac{p}{2 \beta^{2}} & \left(\frac{p}{2}<\beta<p\right)
\end{array}\right\} .
$$

The partial derivative in (2.6) is obviously positive in the case when $\frac{p}{2} \leqq \beta<p$. It is also positive when $p \in \mathbb{N} \backslash\{1,2\}$ and $0<\beta<\frac{p}{2}$, because

$$
p \geqq 2 \quad \Longrightarrow \quad \beta<\frac{p}{2} \leqq p-\sqrt{\frac{p}{2}} \Longrightarrow 1-\frac{p}{2(p-\beta)^{2}}>0 .
$$

Thus, $\alpha(\beta, p)$ is a piecewise strictly increasing function of $\beta$ on the interval $[0, p)$. This means that there exist its piecewise inverse functions. Moreover, it is not difficult to check that $\beta(\alpha, p)$, given by (2.5), is that inverse function.

For $p=1$, it is easy to verify that $\alpha(\beta, 1)$ is continuous on $(0,1)$, concave down on the interval $\left(0, \frac{1}{2}\right)$ and, as stated before, increasing on $\left(\frac{1}{2}, 1\right)$ with $\alpha\left(\frac{1}{2}, 1\right)=0$ and $\alpha(1,1)=1$. Therefore, for its inverse function, we can choose the one that corresponds to $\frac{1}{2} \leqq \beta<1$, that is, the inverse of $\beta-\frac{1}{2}\left(\frac{1-\beta}{\beta}\right)$, which is

$$
\frac{2 \alpha-1+\sqrt{(2 \alpha-1)^{2}+8 p}}{4}=\beta(\alpha, 1)
$$

with domain $[0,1)$.

Remark 2. Theorem 2 is an extension of a sharp result from the earlier work by Srivastava et al. [10] to the case when $0 \leqq \alpha<\frac{p-1}{2}$. Unfortunately, we were not able to prove sharpness of this extension. Thus, the problem of obtaining its sharp versions remains an open problem.

The sharp Marx-Strohhäcker's result for the case when $\frac{p-1}{2} \leqq \alpha<p$ is given by (1.2); for the case when $p=1$ and $0 \leqq \alpha<1$, it is given by (1.3). Comparing the values of $\beta(\alpha, p)$ given by (2.5), $\widehat{\beta}_{1}(\alpha, p)$ given by (1.2) and $\widehat{\beta}_{2}(\alpha)$ given by (1.3), for the case when $\frac{p-1}{2} \leqq \alpha<p$, we obtain the following consequences:

(i) If $p=1$ and $\alpha=0$, then

$$
\widehat{\beta}_{1}(0,1)=\widehat{\beta}_{2}(0)=\beta(0,2)=\frac{1}{2},
$$

which is the Marx-Strohhäcker's result in (1.1);

(ii) If $p=1$ and $0<\alpha<1$, then

$$
\widehat{\beta}_{1}(\alpha, 1)=\widehat{\beta}_{2}(\alpha)>\beta(\alpha, 1) ;
$$


(iii) If $p \in \mathbb{N} \backslash\{1\}$ and $\alpha=\frac{p-1}{2}$, then

$$
\beta(\alpha, p)=\widehat{\beta}_{1}(\alpha, p)=\frac{p}{2},
$$

tha is, the results are equivalent;

(iv) If $p \geqq 2$ and $\frac{p-1}{2}<\alpha<p$, then

$$
\beta(\alpha, p) \neq \widehat{\beta}_{1}(\alpha, p),
$$

that is,

$$
\begin{aligned}
& \qquad \beta(\alpha, p)<\widehat{\beta}_{1}(\alpha, p) \\
& \text { due to the sharpness of } \widehat{\beta}_{1}(\alpha, p) \text {. }
\end{aligned}
$$

\section{A MARX-STROHHÄCKER RESULT OF TYPE II}

In this section, we state and prove a generalization of the implication (1.4) for $p$-valent functions when $p$ is any positive integer.

Theorem 3. Let $p \in \mathbb{N}, 0<\gamma<1$ and

$$
\beta \equiv \beta(\gamma, p)= \begin{cases}p-\frac{1}{2}\left(\frac{\gamma}{1-\gamma}\right) & \left(0<\gamma<\frac{1}{2}\right) \\ p-\frac{1}{2}\left(\frac{1-\gamma}{\gamma}\right) & \left(\frac{1}{2} \leqq \gamma<1\right) .\end{cases}
$$

If $f \in \mathcal{A}_{p}$ and

$$
\Re\left(\frac{z f^{\prime}(z)}{f(z)}\right)>\beta \quad(z \in \mathbb{D}),
$$

then

$$
\Re\left(\frac{f(z)}{z^{p}}\right)>\gamma \quad(z \in \mathbb{D}) .
$$

Proof. The proof of the implication asserted by Theorem 3 runs parallel to that given in the proof of Theorem 1. It makes use of the functions $q(z)$ and $\psi(r, s ; z)$ given by

$$
q(z)=\frac{1}{1-\gamma} \cdot\left(\frac{f(z)}{z^{p}}-\gamma\right) \in \mathscr{H}[1,1]
$$

and

$$
\psi(r, s ; z)=\frac{s(1-\gamma)}{r(1-\gamma)+\gamma}+p,
$$

respectively. Thus, from (3.1), we find that

$$
\psi\left(q(z), z q^{\prime}(z) ; z\right)=\frac{z f^{\prime}(z)}{f(z)} \in \Omega \equiv\{\omega: \Re \omega>\beta\} \quad(z \in \mathbb{D}) .
$$


Furthermore, by the Lemma in Section 1, for proving that

$$
\Re\left(\frac{f(z)}{z^{p}}\right)>\gamma \quad(z \in \mathbb{D}),
$$

that is, that

$$
\Re(q(z))>0 \quad(z \in \mathbb{D}),
$$

it is sufficient to show that

$$
\psi(i x, y ; z)=\frac{y(1-\gamma)}{i x(1-\gamma)+\gamma}+p \notin \Omega
$$

for all real $x, y \leqq-\frac{1+x^{2}}{2}(n=1$ in the Lemma in Section 1$)$ and for all $z \in \mathbb{D}$. Indeed, we have

$$
\begin{aligned}
\Re(\psi(i x, y ; z)) & \leqq \frac{-\left(\frac{1+x^{2}}{2}\right)(1-\gamma) \gamma}{\gamma^{2}+x^{2}(1-\gamma)^{2}}+p \\
& =-\frac{\gamma}{2(1-\gamma)}+\frac{\frac{\gamma(2 \gamma-1)}{2(1-\gamma)}}{\gamma^{2}+x^{2}(1-\gamma)^{2}}+p \equiv \varphi(\gamma, p, x) .
\end{aligned}
$$

Also, from

$$
\frac{\partial}{\partial x} \varphi(\gamma, p, x)=\frac{\gamma(1-\gamma)(1-2 \gamma) x}{\left[\gamma^{2}+x^{2}(1-\gamma)^{2}\right]^{2}}
$$

we find that $\varphi(\gamma, p, x)$ is a piecewise monotone function of $x$ (which increases for $x \geqq 0$ and decreases for $x<0$ when $0<\gamma<\frac{1}{2}$ and which decreases for $x \geqq 0$ and increases for $x<0$ when $\frac{1}{2}<\gamma<1$ ). So, we obtain

$$
\varphi(\gamma, p, x) \leqq\left\{\begin{array}{cc}
\lim _{x \rightarrow+\infty} \varphi(\gamma, p, x)=p-\frac{\gamma}{2(1-\gamma)} & \left(0<\gamma<\frac{1}{2}\right) \\
\varphi(\gamma, p, 0)=p-\frac{1-\gamma}{2 \gamma} & \left(\frac{1}{2} \leqq \gamma<1\right)
\end{array}\right\}=\beta(\gamma, p) .
$$

Remark 3. The value $\beta(\gamma, p)$ is well-defined, that is, $0 \leqq \beta(\gamma, p)<p$. It can be verified by using the fact that, for $0<\gamma<1$, we have

$$
\frac{1}{2(1-\gamma)}>\frac{1}{2} \quad \text { and } \quad \frac{1}{2 \gamma}>\frac{1}{2} \text {. }
$$

The function $\beta(\gamma, p)$, with respect to the variable $\gamma$, is continuous on $(0,1)$ and has the same limit value at the end points of the interval:

$$
\lim _{\gamma \rightarrow 0+} \beta(\gamma, p)=\lim _{\gamma \rightarrow 1-} \beta(\gamma, p)=p .
$$


Also, from

$$
\frac{\partial}{\partial \gamma} \beta(\gamma, p)= \begin{cases}-\frac{1}{2(1-\gamma)^{2}} & \left(0<\gamma<\frac{1}{2}\right) \\ \frac{1}{2 \gamma^{2}} & \left(\frac{1}{2}<\gamma<1\right)\end{cases}
$$

we realize that $\beta(\gamma, p)$ is a strictly decreasing function of the variable $\gamma$ on the interval $\left(0, \frac{1}{2}\right)$ and strictly increasing on the interval $\left(\frac{1}{2}, 1\right)$. Therefore, for the inverse function of $\beta(\gamma, p)$ (over the variable $\gamma$ ), there are two choices: $\frac{2(p-\beta)}{1+2(p-\beta)}$ (which corresponds to $0<\gamma<\frac{1}{2}$ ) and $\frac{1}{1+2(p-\beta)}$ (which corresponds to $\frac{1}{2}<\gamma<1$ ). Since we are interested in the one that gives larger values, for the inverse function, we choose the one that corresponds to $\frac{1}{2}<\gamma<1$ and gives values in the interval $\left(\frac{1}{2}, 1\right)$, instead of the other that gives values in $\left(0, \frac{1}{2}\right)$, that is,

$$
\gamma(\beta, p) \equiv \beta^{-1}(\gamma, p)=\frac{1}{1+2(p-\beta)},
$$

with domain $\left(p-\frac{1}{2}, p\right)$ for the variable $\beta$.

We now rewrite Theorem 3 in the following form.

Theorem 4. Let $p \in \mathbb{N}, p-\frac{1}{2} \leqq \beta<p$ and

$$
\gamma \equiv \gamma(\beta, p)=\frac{1}{1+2(p-\beta)} .
$$

If $f \in \mathcal{A}_{p}$ and

$$
\Re\left(\frac{z f^{\prime}(z)}{f(z)}\right)>\beta \quad(z \in \mathbb{D}),
$$

then

$$
\Re\left(\frac{f(z)}{z^{p}}\right)>\gamma \quad(z \in \mathbb{D}) .
$$

Upon setting $p=1$ in Theorem 3 and Theorem 4, we get the following Corollary.

Corollary 1. Let $f \in \mathcal{A}$. Then the following assertions hold true.

(i) If $0<\gamma<1$, then

$\Re\left(\frac{z f^{\prime}(z)}{f(z)}\right)>\left\{\begin{array}{ll}\frac{3}{2}-\frac{1}{2(1-\gamma)} & \left(0<\gamma<\frac{1}{2}\right) \\ \frac{3}{2}-\frac{1}{2 \gamma} & \left(\frac{1}{2} \leqq \gamma<1\right)\end{array}\right\} \Longrightarrow \Re\left(\frac{f(z)}{z}\right)>\gamma$.

(ii) If $\frac{1}{2} \leqq \beta<1$, then

$$
\Re\left(\frac{z f^{\prime}(z)}{f(z)}\right)>\beta \quad \Longrightarrow \quad \Re\left(\frac{f(z)}{z}\right)>\frac{1}{3-2 \beta} .
$$


All of the above inequalities correspond to the whole unit disk $\mathbb{D}$.

Remark 4. Each of the following observations is worthy of mention here.

(i) For $\gamma=\frac{1}{2}$ in the above Corollary, we get $\beta=\frac{1}{2}$, which is the well-known Marx-Strohhäcker result implied in (1.4). The same conclusion follows for $\beta=\frac{1}{2}$ in the above Corollary.

(ii) Srivastava et al. [10] studied the functions $f \in \mathcal{A}_{p}$ satisfying the following condition:

$$
\begin{gathered}
(1-\lambda)\left(\frac{z f^{(j)}(z)}{f^{(j-1)}(z)}\right)+\lambda\left(1+\frac{z f^{(j+1)}(z)}{f^{(j)}(z)}\right) \prec(p-j+1)\left(\frac{1+A z}{1+B z}\right) \\
(1 \leqq j \leqq p ;-1 \leqq B<A \leqq 1),
\end{gathered}
$$

where the symbol $\prec$ denotes the usual subordination between analytic functions. In fact, Srivastava et al. [10] obtained their result over

$$
\left(\frac{f^{(j-1)}(z)}{z^{p-j+1}}\right)^{v}
$$

but only in the case when $\lambda>0$ (see [10, p. 332, Theorem 5]). Therefore, for $j=A=-B=v=1$, the results presented in this section provide an extension of those given in [10].

\section{A Unified PRESENTATION OF THE MARX-STROHhäCKER TYPE RESUltS}

Theorem 4, when combined with the aforementioned sharp result of Srivastava et al. [10], implies the following theorem.

Theorem 5. Let $p \in \mathbb{N}$ and $\frac{p-1}{2} \leqq \alpha<p$. Suppose also that

$$
\beta \equiv \widehat{\beta}_{1}(\alpha, p)=\frac{p}{{ }_{2} F_{1}\left(1,2(p-\alpha) ; p+1 ; \frac{1}{2}\right)} \geqq p-\frac{1}{2}
$$

and

$$
\gamma \equiv \gamma(\beta, p)=\frac{1}{1+2(p-\beta)}
$$

If $f \in \mathcal{A}_{p}$, then

$$
\Re\left(1+\frac{z f^{\prime \prime}(z)}{f^{\prime}(z)}\right)>\alpha \quad \Longrightarrow \quad \Re\left(\frac{z f^{\prime}(z)}{f(z)}\right)>\beta \quad \Longrightarrow \quad \Re\left(\frac{f(z)}{z^{p}}\right)>\gamma .
$$

All of the above inequalities hold true in the whole unit disk $\mathbb{D}$. 


\section{REFERENCES}

[1] P. L. Duren, Univalent functions, ser. Grundlehren der Mathematischen Wissenschaften [Fundamental Principles of Mathematical Sciences]. Springer-Verlag, New York, 1983, vol. 259.

[2] I. S. Jack, "Functions starlike and convex of order $\alpha$," J. London Math. Soc. (2), vol. 3, pp. 469474, 1971.

[3] T. H. MacGregor, "A subordination for convex functions of order $\alpha$," J. London Math. Soc. (2), vol. 9, pp. 530-536, 1974/75.

[4] A. Marx, "Untersuchungen über schlichte Abbildungen," Math. Ann., vol. 107, no. 1, pp. 40-67, 1933, doi: 10.1007/BF01448878. [Online]. Available: http://dx.doi.org/10.1007/BF01448878

[5] S. S. Miller and P. T. Mocanu, Differential subordinations, ser. Monographs and Textbooks in Pure and Applied Mathematics. Marcel Dekker, Inc., New York, 2000, vol. 225, theory and applications.

[6] M. Nunokawa, A. Ikeda, N. Koike, and Y. Ota, "On multivalently convex and starlike functions," Math. Japon., vol. 49, no. 2, pp. 223-227, 1999.

[7] H. M. Srivastava and A. K. Mishra, "A fractional differintegral operator and its applications to a nested class of multivalent functions with negative coefficients," Adv. Stud. Contemp. Math. (Kyungshang), vol. 7, no. 2, pp. 203-214, 2003.

[8] H. M. Srivastava, A. K. Mishra, and M. K. Das, "A unified operator in fractional calculus and its applications to a nested class of analytic functions with negative coefficients," Complex Variables Theory Appl., vol. 40, no. 2, pp. 119-132, 1999.

[9] H. M. Srivastava and S. Owa, Eds., Current topics in analytic function theory. World Scientific Publishing Co., Inc., River Edge, NJ, 1992. [Online]. Available: http://dx.doi.org/10.1142/1628. doi: $10.1142 / 1628$

[10] H. M. Srivastava, J. Patel, and G. P. Mohapatra, "A certain class of $p$-valently analytic functions," Math. Comput. Modelling, vol. 41, no. 2-3, pp. 321-334, 2005, doi: 10.1016/j.mcm.2003.06.010. [Online]. Available: http://dx.doi.org/10.1016/j.mcm.2003.06.010

[11] E. Strohhäcker, "Beiträge zur Theorie der schlichten Funktionen," Math. Z., vol. 37, no. 1, pp. 356-380, 1933, doi: 10.1007/BF01474580. [Online]. Available: http://dx.doi.org/10.1007/ BF01474580

[12] D. R. Wilken and J. Feng, "A remark on convex and starlike functions," J. London Math. Soc. (2), vol. 21, no. 2, pp. 287-290, 1980, doi: 10.1112/jlms/s2-21.2.287. [Online]. Available: http://dx.doi.org/10.1112/j1ms/s2-21.2.287

Authors' addresses

\section{Nunokawa}

University of Gunma, 798-8 Hoshikuki-cho, Chuou-Ward, Chiba 260-0808, Japan

E-mail address: mamoru-nuno@doctor.nifty.jp

\section{H. M. Srivastava}

Department of Mathematics and Statistics, University of Victoria,, Victoria, British Columbia V8W 3R4, Canada

China Medical University, Taichung 40402, Taiwan, Republic of China

E-mail address: harimsri@math.uvic.ca

\section{N. Tuneski}

Faculty of Mechanical Engineering,, Saints Cyril and Methodius University of Skopje,, Karpoš II b.b., MK-1000 Skopje, Republic of Macedonia

E-mail address: nikola.tuneski@mf.edu.mk 
B. Jolevska-Tuneska

Faculty of Electrical Engineering and Informational Technologies,, Saints Cyril and Methodius University of Skopje,, Karpoš II b.b., MK-1000 Skopje, Republic of Macedonia

E-mail address: bil janaj@feit.ukim.edu.mk 